\title{
Associação entre doenças metabóli- cas e parâmetros hemodinâmicos de filhos obesos e suas mães
}

\section{Association between metabolic and hemodynamic diseases in obese children and their mothers}

Barbara de M M Antunes ${ }^{1 *}$, Paula A Monteiro², Bruno A P de Oliveira ${ }^{3}$, Ricardo R Agostinete ${ }^{4}$, Rômulo A Fernandes ${ }^{5}$, Diego G D Christofaro ${ }^{5}$, Ismael F Freitas Júnior ${ }^{5}$

\begin{abstract}
RESUMO
Modelo do estudo: Estudo original

Objetivo: Verificar possíveis associações entre o perfil hemodinâmico e metabólico de crianças e adolescentes obesos e suas mães. Metodologia: Realizou-se um estudo transversal com 69 crianças e adolescentes obesos, de ambos os sexos, com faixa etária compreendida entre seis e $15(10,6 \pm 3,04)$ anos. Por meio de avaliações específicas foram analisadas as variáveis de composição corporal (massa corporal magra, massa de gordura em quilos e percentual), hemodinâmicas (pressão arterial sistólica e diastólica) e bioquímicas (glicemia, colesterol total e suas frações) dos filhos. Entre as mães foi realizado uma avaliação de composição corporal por meio de antropometria e impedância bioelétrica, aferição de pressão arterial e aplicação de questionário auto referido de doenças. Para o tratamento estatístico foram realizados análise descritiva, teste t de Student independente e análise de associação entre alterações maternas com seus respectivos filhos por meio de teste de Mc Nemar, adotando-se pd" 0,05. Todo o tratamento estatístico foi realizado por meio do software SPSS, 17.0. Resultados: Ser filho de mães diabéticas ou hipertensas associa-se com a presença de alterações nas variáveis de glicemia, pressão arterial sistólica ou diastólica. Quando a mãe apresenta mais de uma variável de risco cardiovascular a sua prole também possui alguma alteração metabólica ou em variáveis dos parâmetros hemodinâmicos (PA). Conclusão: No presente estudo observaram-se associações entre variáveis metabólicas e parâmetros hemodinâmicos entre crianças e adolescentes obesos com suas respectivas mães.
\end{abstract}

Palavras-chave: Criança. Adolescente. Obesidade. Mães. Doenças Metabólicas.

1 Graduada em Educação Física e Mestranda em Ciências da Motricidade- UNESP Rio Claro

2 Mestre em Fisioterapia e Doutoranda em Ciências da Motricidade- UNESP Rio Claro

3 Graduado em Educação Física-UNESP Presidente Prudente. Mestrando - Pós-graduação em Clínica Médica (FMRP-USP), Ribeirão Preto, São Paulo.

4. Graduando em Educação Física- UNESP Presidente Prudente

5. Docente do Departamento de Educação Física UNESP Presidente Prudente
Barbara de Moura Mello Antunes Departamento de Educação Física-UNESP Presidente Prudente, R. Roberto Simonsen, 끈5, Bairro: Vila Santa Helena,

Presidente Prudente/SP-Brasi CEP 19060-900

Endereço eletrônico: bah_tunes@ @otmail.com

Artigo recebido em 09/05/2013 Aprovado para publicação em 04/10/2013 


\section{Introdução}

A obesidade infantil é uma doença crônica de origem multifatorial caracterizada como uma epidemia mundial e importante problema de saúde pública no século XXI, ${ }^{1}$ a qual é observada em países desenvolvidos e em desenvolvimento. ${ }^{2,3}$

O acúmulo excessivo de gordura pode ser decorrente de causas exógenas, tais como fatores ambientais, sociais e culturais ${ }^{4,5}$ e causas endógenas, como influência genética e fatores hormonais. ${ }^{6} \mathrm{O}$ histórico familiar é um aspecto fundamental para a instalação e desenvolvimento da obesidade, observando-se que crianças com pais obesos tem maior probabilidade de serem adultos obesos com alterações metabólicas. ${ }^{7}$ Entretanto, mesmo havendo uma contribuição da genética para esta relação, a Organização Mundial da Saúde (OMS) após análise de vários estudos representativos de diferentes partes do mundo verificou que fatores ambientais, tais como o ambiente familiar, podem ser um dos grandes moderadores dessa relação entre excesso de peso de pais e filhos. ${ }^{8,9}$

Alguns estudos relataram diversas contribuições genéticas dos pais sobre o estado nutricional e metabólico dos filhos, principalmente a significante influência materna sobre o desenvolvimento da criança ao longo da vida, enfatizando não só o estado nutricional no período pré-gestacional e ao longo da gravidez, como também a nutrição da criança nos primeiros anos de vida. ${ }^{10,11}$

Estudo realizado por Frutuoso et $\mathrm{al}^{12}$ aponta que excesso de adiposidade nos pais, principalmente da mãe, representa um dos fatores de risco mais importantes para o desenvolvimento da obesidade no filho e pode estar associada com outras alterações metabólicas. É bem estabelecido que o histórico familiar para algumas alterações hemodinâmicas, como a hipertensão arterial, é um forte fator desencadeante para o desenvolvimento de hipertensão nos filhos e que esta influência é potencializada quando ambos os progenitores são hipertensos. ${ }^{13}$

Desta forma, o objetivo do presente estudo foi verificar possíveis associações entre o perfil hemodinâmico (valores de pressão arterial sistêmica) e metabólico de jovens obesos com suas respectivas mães.

\section{Métodos}

O cálculo amostral foi baseado em correlação linear. Adotou-se $\mathrm{r}=0.35$ (arbitrário devido à carência de informações sobre os diferentes indicadores analisados), poder de $80 \%$ e significância de $5 \%$. Este cálculo indicou a necessidade de avaliar ao menos 63 jovens. O presente estudo de caráter transversal foi composto por 69 crianças e adolescentes obesos (ambos os sexos), com faixa etária entre seis e 16 anos $(10,6 \pm 3,04)$ e participantes de um projeto de extensão universitária, denominado "Super-Ação" que proporciona tratamento gratuito, não farmacológico e multidisciplinar à obesidade infantil para a população pediátrica obesa de Presidente Prudente e região após a divulgação do referido projeto na mídia local. $\mathrm{O}$ mesmo foi desenvolvido no Departamento de Educação Física da Universidade Estadual Paulista-UNESP, Campus de Presidente Prudente. Para ingresso no referido programa são critérios de inclusão: (a) o jovem ser classificado obeso pelo Índice de Massa Corporal (IMC), seguindo critério publicado por Cole et al. ${ }^{14}$; (b) ter entre seis e 16 anos completos, na data da avaliação; (c) não apresentar nenhum problema de ordem clínica que impeça a prática de atividades físicas; (d) os pais ou responsáveis legais assinarem o termo de consentimento formal para participação, sendo excluído do referente estudo os indivíduos que não preenchiam todos os critérios de inclusão mencionados. Esse projeto foi aprovado pelo Comitê de Ética em Pesquisa da UNESP de Presidente Prudente (protocolo 07/2009).

\section{Coleta dos dados dos filhos}

A massa corporal foi aferida com a utilização de uma balança eletrônica da marca Filizola, com precisão de $0,1 \mathrm{~kg}$ e capacidade máxima de $180 \mathrm{~kg}$, de acordo com a metodologia proposta por Lohman et al. ${ }^{15}$ A estatura foi aferida com a utilização de um estadiômetro fixo, marca Sanny, com precisão de $0,1 \mathrm{~cm}$ e extensão máxima de dois metros, de acordo com a metodologia proposta por Lohman et al. ${ }^{15}$ Por meio destas medições foi calculado o IMC.

Para a análise da composição corporal e da distribuição da gordura corporal foi empregada a técnica da absortiometria de raios-X de dupla energia (DEXA), utilizando-se o equipamento modelo GE Lunar - DPXNT e software 4.7. O método estima a composição corporal fracionando o corpo em três compartimentos anatômicos: massa livre de gordura (massa corporal magra), massa de gordura e conteúdo mineral ósseo. Esta técnica permite estimar a composição corporal no todo e por segmento corporal, desta forma, foi estimada a composição corporal do tronco e apendicular 
e essas medidas foram expressas em gramas (g) e em percentual do total. A dose de radiação que o avaliado recebeu foi menor do que $0,05 \mathrm{mrem}^{16}$, ou seja, equivale a 50 vezes menos a realização de um exame de raio $\mathrm{X}$. O exame tem a duração de aproximadamente 15 minutos e após o posicionamento do avaliado no aparelho, o mesmo permaneceu imóvel e em decúbito dorsal durante todo o exame, e ao término da leitura, os resultados foram transmitidos a um computador interligado ao aparelho e os dados de Massa Corporal Magra, Gordura corporal em kg e em percentual, conteúdo e densidade mineral óssea foram posteriormente analisados.

A aferição da Pressão Arterial Sistólica (PAS) e Diastólica (PAD), bem como da frequência cardíaca, foi realizada com esfignomanômetro automático digital (marca Omron Healthcare, Inc., Intellisense, modelo HEM 742 INT, Bannockburn, Illinois, USA) com manguitos de tamanho apropriado à circunferência do braço dos avaliados. As medidas foram feitas após repouso de, aproximadamente, 15 minutos, na posição supina e obtida no braço direito. Foram adotados os pontos de corte recomendados pela VI Diretrizes Brasileiras de Hipertensã $o^{17}$, sendo considerados portadores de hipertensão arterial todos os indivíduos que apresentarem valores de PAS e ou PAD acima do percentil 95 recomendado para sua respectiva idade e estatura.

A coleta de sangue foi realizada em laboratório particular onde os indivíduos foram avaliados após jejum de 12 horas. A coleta foi realizada em tubo a vácuo com gel separador sem anticoagulante. Após a coleta, o sangue foi centrifugado por 10 minutos a 3.000 rpm para separar o soro dos demais componentes do sangue, sendo o soro utilizado para as análises de glicose e perfil lipídio. Para dosagem da glicemia, triglicérides, colesterol total e suas frações (lipoproteína de alta [HDL-Colesterol] e baixa densidade [LDLColesterol]) foi utilizado um kit enzimático colorimétrico processado em um aparelho Autohumalyzer A5. As anormalidades dos valores das variáveis bioquímicas foram definidas de acordo com as referências propostas pela III Diretrizes Brasileiras sobre Dislipidemias e diretriz sobre prevenção e aterosclerose ${ }^{18}$, e Federação Internacional de Diabetes. ${ }^{19}$

Todas as avaliações supracitadas foram realizadas anteriormente ao ingresso dos participantes ao tratamento não farmacológico, composto basicamente por exercício físico e vivências nutricionais e psicológicas, como ferramenta avaliativa do estado nutricional dos mesmos.

\section{Coleta dos dados das mães}

A metodologia aplicada para mensuração da estatura e massa corporal foi semelhante à empregada para coleta das mesmas variáveis dos filhos e utilizando-se dos mesmos equipamentos. Para a estimativa da composição corporal pela impedância bioelétrica (BIA), utilizou-se o aparelho BIA analyzer - $101 Q$ (marca RJL Systems, Detroit) com freqüência simples, que fornece os valores de resistência e reatância, em Ohms (Ù). Por meio da equação elaborada por Sun et al. ${ }^{20}$, foi possível estimar a massa livre de gordura (massa magra) e, consequentemente, a massa de gordura (MG) e percentual de gordura corporal (\%GC). A técnica foi conduzida como na literatura. ${ }^{21}$.

Para a aferição da frequência cardíaca, PAS e PAD aplicou-se metodologia similar à realizada nos filhos utilizando-se manguitos de tamanho apropriado à circunferência do braço das mães. Foram consideradas hipertensas mulheres com pressão sistólica igual ou superior a $130 \mathrm{mmHg}$ e a pressão diastólica igual ou superior a $85 \mathrm{mmHg}$ como proposto pela VI Diretrizes Brasileira de Hipertensão Arterial. ${ }^{17}$

As informações sobre alterações metabólicas, tais como Diabetes Mellitus, Dislipidemia e Hipertensão foram coletadas por meio de questionário auto referido, composto por questões fechadas, onde as mesmas foram orientadas a responderem sobre sua saúde assinalando a presença ou a ausência das doenças supracitadas, de acordo com diagnóstico médico prévio, bem como o tratamento e ingestão de medicamento para as referidas alterações. O preenchimento do questionário auto referido, bem como as avaliações de composição corporal e hemodinâmicas, foram realizadas no mesmo dia de coleta de medidas de seus respectivos filhos compondo o inquérito avaliativo antecedente ao tratamento.

\section{Análise Estatística}

Para testar a hipótese de normalidade dos dados aplicou-se o teste de Shapiro Wilk observando-se distribuição normal das variáveis analisadas. Posteriormente, foram utilizadas formas de estatísticas descritivas: média e desvio-padrão. O coeficiente de correlação de Spearman foi empregado com o intuído de analisar a relação entre os postos ocupados por filhos em mães em seus respectivos grupos. Para analisar a presença ou ausência de associação entre doenças de mães e filhos, e, influência de fatores de risco existente de mães sobre os filhos utilizou-se o teste de McNemar adotando significância estatística menor que $5 \%(\mathrm{p}<0,05)$. 


\section{Resultados}

A amostra do presente estudo foi composta, em sua totalidade, por crianças e adolescentes obesos que apresentaram prevalências elevadas para algumas alterações metabólicas, tais como, pressão arterial $(30,9 \%)$, colesterol total $(18,8 \%)$, HDL $(15,9 \%)$ e LDL $(20,6 \%)$.

Quando ajustado aos gêneros os meninos apresentaram, para a maioria das variáveis, maiores valores absolutos de medidas quando comparados às meninas. Entretanto, só houve significância estatística para $\% \mathrm{GC}(\mathrm{p}=0,008), \mathrm{MCM}$ em quilos $(\mathrm{p}=0,031)$, glicemia $(\mathrm{p}=0,018)$ e \% Gordura de tronco $(\mathrm{p}<0,001)$. Quando ajustado entre crianças (seis a nove anos) e adolescentes (10 a 15 anos) houve diferença entre as medidas de peso $(\mathrm{p}<0,001)$, estatura $(\mathrm{p}<0,001)$, PAS ( $\mathrm{p}=0,003), \mathrm{MG}$ em quilos ( $\mathrm{p}<0,001$ ), MCM em quilos $(\mathrm{p}<0,001)$, Colesterol total $(\mathrm{p}=0,027)$ e HDL $(\mathrm{p}=0,049)$. Na Tabela 1 e Tabela 2 são apresentadas as descrições da amostra compostas por crianças e adolescentes obesos e de suas mães, e de acordo com o gênero, respectivamente.
São apresentadas na Tabela 3 as correlações existentes entre as variáveis hemodinâmicas (PA e FC), metabólicas e de composição corporal entre mães e filhos adotando significância de 5\%. Observa-se que, principalmente, as medidas hemodinâmicas maternas correlacionam-se com as alterações dos filhos.

$\mathrm{Na}$ Figura 1 está ilustrada, em valores relativos, a associação entre fatores de risco de mães e filhos. Foi possível observar maior ocorrência de pressão arterial elevada e glicemia alterada em jovens filhos de mães hipertensas $(p=0,035)$ e diabéticas $(p=$ $0,016)$, respectivamente.

\section{Discussão}

Em nossa casuística observou-se que $95,7 \%$ das mães das crianças e dos adolescentes obesos estão com excesso de peso e percentual de gordura acima dos valores propostos como ideais, caracterizando-se como mães obesas. Estudos têm indicado que o ganho de peso excessivo ao longo da gestação pode ser a causa da obesidade em muitas mulheres e que pode contribuir para o aumento do peso no filho. Caracte-

Tabela 1

Caracterização das variáveis metabólicas e níveis pressóricos dos filhos (Presidente Prudente - SP, 2012).

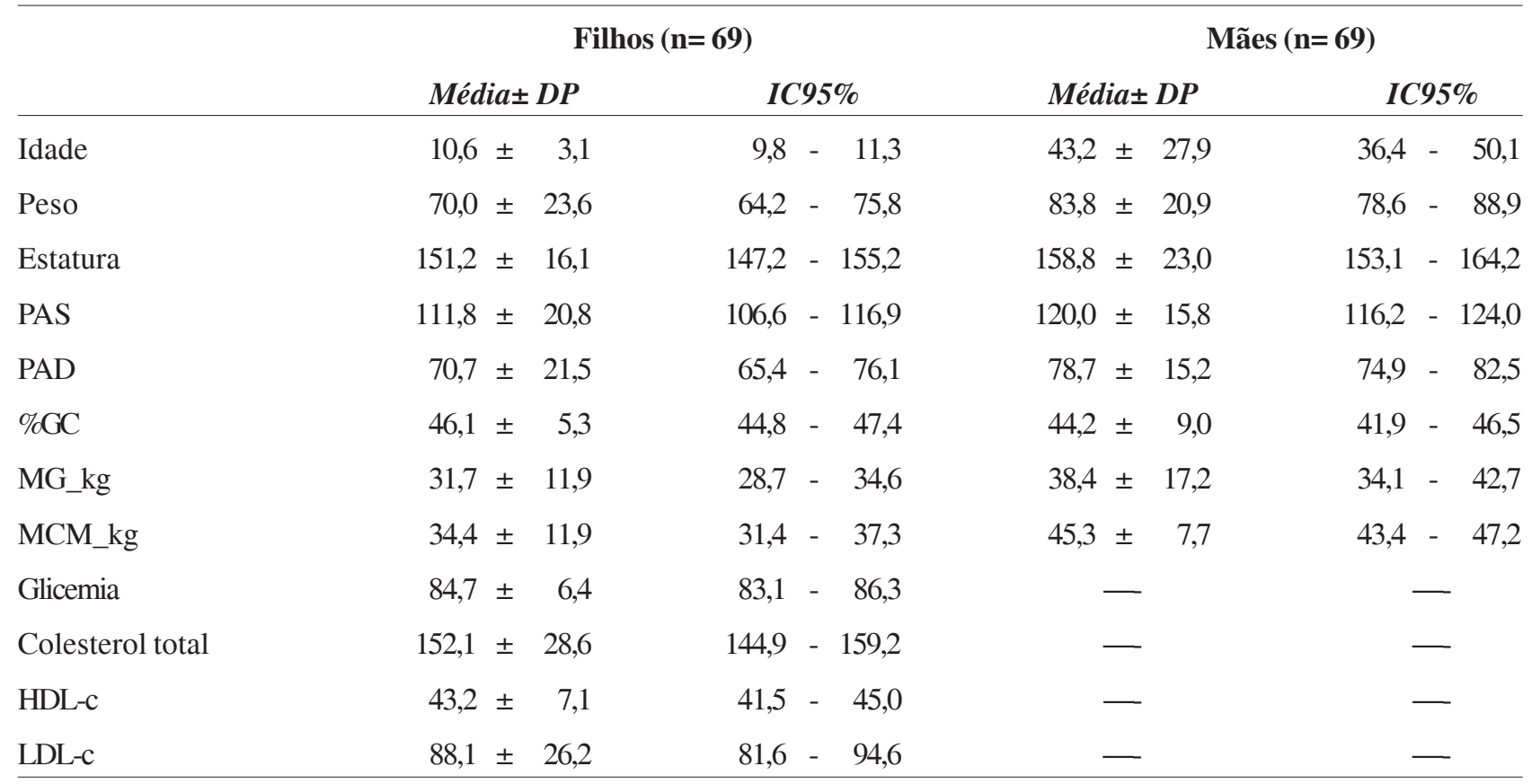

$\mathrm{DP}=$ desvio-padrão; $\mathrm{PAS}=$ pressão arterial sistólica; $\mathrm{PAD}=$ pressão arterial diastólica; \%GC= percentual de gordura corporal; $\mathrm{MG} \_\mathrm{kg}=$ massa de gordura em quilos; MCM_kg=massa corporal magra em quilos; HDL-C= high density lipoprotein; LDL-C= low density lipoprotein; IC95\%= intervalo de confiança de $95 \%$. 
Tabela 2

Descrição da amostra de acordo com o gênero (Presidente Prudente - SP, 2012).

\begin{tabular}{|c|c|c|c|c|c|c|c|c|}
\hline \multirow[b]{3}{*}{ Idade } & \multicolumn{2}{|c|}{ Meninos $(n=34)$} & \multicolumn{2}{|c|}{ Meninas $(n=35)$} & \multirow[b]{2}{*}{$p$} & & & \\
\hline & Média \pm & DP & Média \pm & DP & & \multicolumn{3}{|c|}{ IC95\% } \\
\hline & $10,9 \pm$ & 3,3 & $10,3 \pm$ & 2,7 & 0,447 & $-0,9$ & - & 2,0 \\
\hline Peso & $74,6 \pm$ & 25,8 & $65,5 \pm$ & 19,8 & 0,110 & $-2,1$ & - & 20,3 \\
\hline Estatura & $154,2 \pm$ & 17,7 & $148,5 \pm$ & 13,6 & 0,145 & $-2,0$ & - & 13,4 \\
\hline PAS & $115,3 \pm$ & 18,0 & $106,6 \pm$ & 22,3 & 0,079 & $-1,0$ & - & 18,5 \\
\hline $\mathrm{PAD}$ & $71,7 \pm$ & 27,4 & $69,4 \pm$ & 11,9 & 0,642 & $-7,7$ & - & 12,4 \\
\hline$\% \mathrm{GC}$ & $44,4 \pm$ & 5,6 & $47,7 \pm$ & 4,4 & $0,008^{*}$ & $-5,7$ & - & $-0,9$ \\
\hline MG_kg & $32,1 \pm$ & 13,2 & $30,5 \pm$ & 10,1 & 0,496 & $-3,7$ & - & 7,6 \\
\hline MCM_kg & $37,3 \pm$ & 12,9 & $31,3 \pm$ & 9,4 & $0,031 *$ & 0,5 & - & 11,4 \\
\hline Glicemia & $86,3 \pm$ & 7,1 & $82,0 \pm$ & 7,5 & $0,018^{*}$ & 0,8 & - & 7,8 \\
\hline Colesterol total & $149,1 \pm$ & 25,1 & $154,3 \pm$ & 30,6 & 0,439 & $-18,7$ & - & 8,2 \\
\hline HDL-c & $43,9 \pm$ & 6,9 & $42,6 \pm$ & 7,7 & 0,471 & $-2,2$ & - & 4,8 \\
\hline LDL-c & $84,1 \pm$ & 23,2 & $90,4 \pm$ & 28,7 & 0,318 & $-18,9$ & - & 6,2 \\
\hline
\end{tabular}

Tabela 3

Relação entre os fatores metabólicos, pressóricos e de composição corporal entre mães e filhos (Presidente Prudente - SP, 2012).

\begin{tabular}{|c|c|c|c|c|c|c|}
\hline \multicolumn{7}{|c|}{ Variáveis Maternas } \\
\hline Filhos & $P A S$ & $P A D$ & Hipertensão & $M G$ & $M C M$ & $\% G C$ \\
\hline$P A S$ & $\begin{array}{c}0,231 \\
(p=0,056)\end{array}$ & & $\begin{array}{c}0,213 \\
(p=0,079)\end{array}$ & & & \\
\hline$P A D$ & & $\begin{array}{c}0,082 \\
(p=0,502)\end{array}$ & $\begin{array}{c}0,274 * \\
(p=0,023)\end{array}$ & & & \\
\hline$M G$ & & & & $\begin{array}{c}0,013 \\
(p=0,915)\end{array}$ & & \\
\hline$M C M$ & & & & & $\begin{array}{c}0,140 \\
(p=0,252)\end{array}$ & \\
\hline$\% G C$ & & & & & & $\begin{array}{c}0,053 \\
(p=0,668)\end{array}$ \\
\hline
\end{tabular}

$\mathrm{PAS}=$ pressão arterial sistólica; $\mathrm{PAD}=$ pressão arterial diastólica; $\mathrm{MCM}=$ massa corporal magra em quilos; $M G=$ massa de gordura em quilos; \%GC=percentual de gordura corporal; $p=$ significância estatística. 




Figura 1. Associação de fatores de risco entre mães e filhos. (Presidente Prudente - SP, 2012).

rísticas como idade, escolaridade, intervalo entre gestações ${ }^{22,23}$, e principalmente, hábitos nutricionais e prática reduzida de exercícios físicos apresentam-se como fatores que podem contribuir para a instalação do excesso de peso nas mães, podendo contribuir para o excesso de peso em seus filhos. ${ }^{24}$

Alguns trabalhos sugerem que os maus hábitos maternos durante a gestação são determinantes para a instalação de complicações metabólicas no filho. $\mathrm{O}$ excesso de peso da mãe na fase gestacional pode proporcionar um aumento nas concentrações plasmáticas de ácidos graxos livres e de glicose o que pode aumentar a chance desses filhos terem excesso de peso futuramente. ${ }^{25}$ Além disso, desde os primeiros anos de vida até a fase adulta, o comportamento materno mostra-se capaz de contribuir positivamente no desenvolvimento da criança e de sua composição corporal, podendo ser este último aspecto um fator independente para o desenvolvimento de complicações osteoarticulares, psicológicas, cardiovasculares, respiratórias e metabólicas. ${ }^{26,27}$

Ressalta-se que fatores genéticos não são os únicos contribuintes para a presença de alterações metabólicas e pressóricas em crianças, pois há diversos fatores de risco externos, tais como hábitos alimentares inadequados, sedentarismo, condições socioeconômicas e sobrepeso/obesidade dos pais, que também contribuem diretamente para o estado nutricional e o desenvolvimento de doenças em crianças e adolescentes. Estudos evidenciam que o contexto familiar (estilo de vida e hábitos cotidianos) influencia na formação dos hábitos alimentares e prática de atividade física de crianças, ${ }^{28,29,30}$ e desta forma, indicam que hábitos de ambientes familiares obesogênicos somados à hereditariedade compõem importantes fatores para o desenvolvimento da obesidade e de comorbidades associadas a doença. ${ }^{31}$

No presente estudo, observou-se associação entre elevação dos níveis de pressão arterial materna e entre os filhos, resultado este que não é coincidente com os resultados de Mendes et al..$^{32}$ que não encontrou associação estatisticamente significante entre os níveis pressóricos dos pais com seus respectivos filhos. Porém, no presente estudo realizaram-se medidas diretas da pressão arterial não apenas nos adolescentes, mas também em todas as mães, diferentemente do estudo de Mendes et al. ${ }^{32}$ que considerou apenas aqueles pais que recebiam tratamento farmacológico para hipertensão arterial.

Corroborando com nossos achados Fermino et al. ${ }^{33}$ em um estudo que objetivou observar possíveis associações entre os valores de pressão arterial 164 progenitores e 203 descendentes encontram uma correlação significativa. $\mathrm{O}$ estudo mencionado realizou análise de heritabilidade verificando que os fatores genéticos podem explicar em até $45 \%$ a associação dos valores de pressão arterial.

$\mathrm{Na}$ referida pesquisa observou-se associação entre diabetes materno e valores elevados de glicemia entre os jovens. Estudos indicam que o histórico familiar de hiperglicemia ou de diabetes mellitus em um dos progenitores é uma variável de risco para o 
desenvolvimento da mesma doença e/ou alterações glicêmicas na prole. Entretanto esta influência parece ser mais forte quando advinda de sua progenitora (mãe) em decorrência de influxos metabólicos que podem ocorrer na fase intra-uterina, bem como, em virtude de sua maior responsabilidade alimentar, contato e educação com o filho, sobre a ingestão alimentar e os comportamentos cotidianos. ${ }^{34}$ De fato, sabe-se que a macrossomia é um desfecho fortemente associado ao diabetes gestacional, bem como, que o ganho de peso da mãe na gestação está associado à macrossomia no filho, contribuindo dessa forma para a instalação de elevados valores de glicemia nesses jovens. ${ }^{35}$

A principal limitação deste estudo está no fato de ser um estudo observacional transversal, o que limita as análises de causalidade. Assim, novas investigações de delineamento longitudinal sobre a temática devem ser realizadas a fim de se obter melhor compreensão sobre essa complexa interação, sob a corre- ção de outros cofatores: controle de ingestão alimentar de mães e filhos, comportamento materno ao longo da gestão e pós- gestação, alterações metabólicas e hemodinâmicas durante a gestação, entre outros. Porém ressalta-se como gerador de hipóteses a sugestão de relação entre variáveis maternas e filhos obesos, fornecendo informações úteis ao combate à epidemia do excesso de peso e seus possíveis fatores de risco.

Sendo assim, conclui-se com o presente estudo que há associações entre variáveis metabólicas e níveis de pressão arterial sistêmica de jovens obesos e suas respectivas mães, observando-se relação positiva entre hipertensão materna e alteração da PAD do filho, além de verificar que crianças que possuem pressão arterial elevada e glicemia alterada são, respectivamente, filhos de mães hipertensas e diabéticas. Ações de promoção da saúde que visem à diminuição do excesso de peso e aumento da prática de atividade física no ambiente familiar devem ser incentivadas.

\begin{abstract}
Study Model: Original Study

Objective: To analyze possible associations between hemodynamic and metabolic profile of obese children and adolescents and their mothers. Methods: The study had longitudinal design with 69 children and adolescents, aged between six and $15(10,6 \pm 3,04)$ years old. Specific assessments to body composition (fat free mass, fat mass and percentage fat), blood pressure (blood pressure systolic and diastolic) and biochemical analysis of blood plasma (glucose, total cholesterol and its fractions) of children were analyzed. Among the mothers was carried out an assessment of body composition by anthropometry and bioelectrical impedance, blood pressure and application of inquiry self-report diseases. To treatment statistic were applied descriptive analyze, independent Student's test and test for association between maternal disorders with their children by Mc Nemar test, adopting pd" 0,05. Statistical analyses were performed using SPSS software version 17.0 (Statistical Package for Social Science, SPSS Inc,Illinois, USA). Results: Diabetic or hypertensive mothers contribute to the presence or absence of disorders in glucose, blood pressure systolic or diastolic in their children, as well as, when mothers has more than one cardiovascular risk variable their children had some disorder metabolic or hemodynamic too. Conclusion: The present study notes association between metabolic and hemodynamic variables of children and adolescents with their mothers.
\end{abstract}

Keywords: Child. Adolescent. Obesity. Mothers. Metabolic Diseases.

\section{Referências}

1. Hills AP, Andersen LB, Byrne NM. Physical activity and obesity in children. Br J Sports Med. 2011; 866-70.

2. Lamounier JA. Transição epidemiológica nutricional em crianças e adolescentes argentinos de áreas carentes. Rev Paul Pediatr. 2009; 27: 124-6.

3. Instituto Brasileiro de Geografia e Estatística. Apresenta informações sobre antropométrica e análise do estado nutricional de crianças e adolescentes no Brasil [homepage na Internet]. 2006 [acesso em 2012 Jun 6]. Disponível em: http:// www.ibge.org.br
4. Simões NP, Raifur MS. Análise da Prevalência de Sobrepeso e Obesidade em Adolescentes Matriculados na Rede Pública de Ensino de Ivaí (PR). Rev Bras Ter e Saúde. 2011; 1: 83-94.

5. Sapatera, MLR, Pandini EV. Obesidade na adolescência. Lecturas: Educación Física y Deportes - Revista Digital 2005; 10(85).

6. Landeiro FM, Quarantini LC. Obesidade: controle neural e hormonal do comportamento alimentar. Rev Ciênc Méd Biol. 2011; 10: 236-45.

7. Miranda AAN, Navarro F. A prevenção e o tratamento da obesidade durante infância: uma opção eficaz para reduzir a prevalência desta patologia. Revista Brasileira de Obesidade, Nutrição e Emagrecimento. 2008; 2:313-23. 
8. [No authors listed] Diet, nutrition and the prevention of chronic diseases. W.H.O. Tech Rep Ser. . 2003;916:i-viii, 1-149, backcover.

9. Swinburn BA, Caterson I, Seidell JC, James WP. Diet, nutrition and the prevention of excess weight gain and obesity. Public Health Nutr.. 2004;7(1A):123-46.

10. Vendruscolo JF, Bolzan GM, Crestani AH, Souza APR, Moraes $A B$. A relação entre o aleitamento, transição alimentar e os indicadores de risco para o desenvolvimento infantil. Distúrb Comun.. 2012; 24: 41-52.

11. Kivima"ki M, Lawlor DA, Smith GD, Elovainio M, Jokela M, Keltikangas- Ja"rvinen L, et al. Substantial intergenerational increases in body mass index are not explained by the fetal overnutrition hypothesis: the Cardiovascular Risk in Young Finns Study. Am J Clin Nutr.. 2007; 86:1509-14.

12. Frutuoso MFP, Bovi TG and Gambardella AMD. Adiposidade em adolescentes e obesidade materna. Rev. Nutr.[on line] 2011; 24: 5-15.

13. Wang NY, Young JH, Meoni LA, Ford DE, Erlinger TP, Klag MJ. Blood pressure change and risk of hypertension associated with parental hypertension: The Johns Hopkins Precursors Study. Arch Intern Med. 2008; 168: 643-48.

14. Cole TJ, Bellizzi MC, Flegal KM, Dietz WH. Establishing a standard definition for child overweight and obesity worldwide: international survey. BMJ. 2000; 320:1240-3.

15. Lohman TG, Roche AF, Martorell R. Anthropometric Standardization Reference Manual. Champaign, Illinois: Human Kinetics 1988; 177.

16. Laskey MA, Crisp AJ, Cole TJ, Compston JE. Comparison of the effect of different reference data on Lunar DPX and Hologic QDR-1000 dual-energy X-ray absorptiometers. Br J Radiol. 1992; 65:1124-9.

17. Sociedade Brasileira de Cardiologia. VI Diretrizes Brasileira de Hipertensão Arterial. Rev Bras Hipertens. 2010; 13 :1-68.

18. III Diretrizes Brasileiras Sobre Dislipidemias e Diretriz de Prevenção da Aterosclerose do Departamento de Aterosclerose da Sociedade Brasileira de Cardiologia. Arq Bras Cardiol. 2001; 77: 1-48.

19. International Diabetes Federation. The IDF Consensus Worldwide Definition of the Metabolic Syndrome in Childrens and Adolescents. [homepage da Internet]

20. Sun SS, Chumlea WC, Heymsfield SB, Lukaski HC, Schoeller $D$, Friedl $K$ et al. Development of bioelectrical impedance analysis prediction equations for body composition with the use of a multicomponent model for use in epidemiologic surveys. Am J Clin Nutr.. 2003; 77:331-40.

21. Heyward VH, Stolarczyk LM. Applied body composition assessment. Champaign, IL: Human Kinetics. 1996.

22. Leopércio W, Gigliotti A. Tabagismo e suas peculiaridades durante a gestação: uma revisão crítica. J Bras Pneumol. 2004; 30: 176-85
23. Stulbach TE, Benicio MHA, Andreazza R, Kono S. Determinantes do ganho ponderal excessivo durante a gestação em serviço publico de pré-natal de baixo risco. Rev Bras Epidemiol. 2007; 10: 99-108.

24. Nascimento SL, Surita FGC, Parpinelli MA, Cecatti JG. Exercício físico no ganho de peso e resultados perinatais em gestantes com sobrepeso e obesidade: uma revisão sistemática de ensaios clínicos. Cad Saúde Pública. 2011; 27:407-16.

25. Davey Smith G, Steer C, Leary S, Ness A.Is there an intrauterine influence on obesity? Evidence from parent child associations in the Avon Longitudinal Study of Parents and Children (ALSPAC). Arch Dis Child. 2007; 92:876-80.

26. Peres SHCS, Goya S, Sant'Anna RMF, Silva HM, Peres ACS, Silva RPR, et al. Prevalência de sobrepeso e obesidade e fatores associados em adolescentes na região centro-oeste do estado de São Paulo. Cien Saude Colet. 2010; 15: 3175-84.

27. Gigante DPB, Moura EC, Sardinha LMV. Prevalence of overweight and obesity and associated factors. Rev Saúde Pública 2009; 43: 83-9.

28. Bernardo, CO, Pudla, KJ, Longo, GZ and Vasconcelos, FAG de. Fatores associados ao estado nutricional de escolares de 7 a 10 anos: aspectos sociodemográficos, de consumo alimentar e estado nutricional dos pais. Rev. bras. epidemiol. [online]. 2012; 15: 651-61

29. Bernardo CO, Fernandes PS, Campos RMMB, Adami F, Vasconcelos FAG. Associação entre o índice de massa corporal de pais e de escolares de 7 a 14 anos de Florianópolis, SC, Brasil. Rev Bras Saúde Matern Infant. 2010; 10: 183-90.

30. Enes CC, Slater B. Obesidade na adolescência e seus principais fatores determinantes. Rev Bras Epidemiol. 2010; 13: 163-71.

31. Benedet J, Assis MAA de; Calvo MCM and Andrade DF de. Excesso de peso em adolescentes: explorando potenciais fatores de risco. Rev Paul Pediatr. [online]. 2013, 31: 17281

32. Mendes MJFL, Alves JGB, Alves AVS, Siqueira PP, Freire EFC. Associação de fatores de risco para doenças cardiovasculares em adolescentes e seus pais. Rev. Bras. Saúde Matern Infant. 2006; 6: 49-54.

33. Fermino RC, Seabra A, Garganta R, Maia JÁ. Genetic Factors in Familial Aggregation of Blood Pressure of Portuguese Nuclear Families. Arq Bras Cardiol 2009; 92: 209-15.

34. Abbasi A, Corpeleijn E, Van der Schouw RP, Stolk AMW, Spijkerman DL van der Schouw AG, et al. Maternal and paternal transmission of type 2 diabetes: influence of diet, lifestyle and adiposity. Int J Med. 2011; 270: 388-96.

35. Costa BM, Paulinelli RR, Barbosa MA. Association between maternal and fetal weight gain: cohort study. Sao Paulo Med J. 2012; 130:242-7. 